Revista Arbitrada Interdisciplinaria KOINONIA

Año V. Vol V. N9. Enero - Junio 2020

Hecho el depósito de Ley: FA2016000010 ISSN: 2542-3088

FUNDACIÓN KOINONIA (F.K). Santa Ana de Coro. Venezuela.

Carlos Julio Bastidas Martínez; Roberth Olmedo Zambrano Santos

http://dx.doi.org/10.35381/r.k.v5i9.580

\title{
El duelo familiar y el desarrollo emocional en los estudiantes
}

\section{Family grieving and emotional development in students}

\author{
Carlos Julio Bastidas-Martínez \\ carlos2210@yahoo.com \\ Pontificia Universidad Católica del Ecuador, Extensión Manabí, Portoviejo \\ Ecuador \\ https://orcid.org/0000-0001-8519-0664 \\ Roberth Olmedo Zambrano-Santos \\ rzambranosantos@yahoo.es \\ Pontificia Universidad Católica del Ecuador, Extensión Manabí, Portoviejo \\ Universidad Estatal del Sur de Manabí, Portoviejo \\ Instituto Superior Tecnológico Portoviejo, Portoviejo \\ Ecuador \\ https://orcid.org/0000-0002-4072-4738
}

Recibido: 1 de noviembre de 2019

Revisado: 15 de noviembre de 2019

Aprobado: 15 de diciembre de 2019

Publicado: 20 de enero de 2020

\section{RESUMEN}

La investigación tuvo como objetivo general determinar la relación entre el duelo familiar y el desarrollo emocional en los estudiantes de educación general básica superior y bachillerato general unificado de la Unidad Educativa Fiscal Cascol, de la Parroquia Cascol del Cantón Paján, provincia de Manabí, Ecuador. Metodológicamente se abordó desde un tipo correlacional transeccional, aplicándose un instrumento tipo Likert a una población de 200 estudiantes. El resultado de -,232 indica una correlación de Pearson negativa débil, lo cual implica una relación inversamente proporcional, es decir que el desarrollo emocional de los estudiantes incide directamente en el afrontamiento del duelo familiar. Al existir correlación significativa en el nivel 0,01 se acepta la hipótesis afirmativa y se rechaza la nula. Siendo necesaria una adecuada intervención por parte de los docentes, padres y representantes en apoyar a los estudiantes a superar el duelo.

Descriptores: Psicología de la educación; rendimiento escolar; eficacia del docente; familia. (Palabras tomadas del Tesauro UNESCO). 
Revista Arbitrada Interdisciplinaria KOINONIA

Año V. Vol V. N9. Enero - Junio 2020

Hecho el depósito de Ley: FA2016000010

ISSN: 2542-3088

FUNDACIÓN KOINONIA (F.K). Santa Ana de Coro. Venezuela.

Carlos Julio Bastidas Martínez; Roberth Olmedo Zambrano Santos

\begin{abstract}
The general objective of the research was to determine the relationship between family grieving and emotional development in general basic, high school and higher education students at Fiscal Cascol Educational Unit located in Cascol Parish, Paján Canton, Manabí Province, Ecuador. Methodologically, it was approached from a correlational type, applying a Likert-type instrument to a population of 200 students. The result of ,232 indicates a weak negative Pearson correlation, which implies an inversely proportional relationship, that is to say, that the emotional development of the students directly affects the confrontation of family grieving. When there is a significant correlation at level 0.01, the affirmative hypothesis is accepted and the null is rejected. It is necessary an adequate intervention by teachers, parents and representatives to support students to overcome the duel.
\end{abstract}

Descriptors: Educational psychology; academic achievement; teacher effectiveness; family. (Words taken from the UNESCO Thesaurus).

\title{
INTRODUCCIÓN
}

Es común en el ser humano, realizar la pregunta sobre ¿qué se entiende por duelo? Surgen respuestas como: fallecimiento, vacío, ausencia, dolor, angustia, desesperación, soledad, miedo, desolación, desazón, tristeza, abatimiento, pesadumbre, pena, tormento, remordimiento, llanto, sufrimiento o consternación. Estas emociones, vivencias, son las responsable de la forma de ser, porque las personas son el resultado de su crecimiento, desarrollo y son determinantes para tener una cosmovisión del entorno que les rodea.

La mayoría de las personas se recupera en un periodo de tiempo relativamente corto que oscila entre los 2 y 3 años. Existen factores que pueden condicionar positiva o negativamente este proceso, incrementando o suavizando la intensidad y duración del mismo y, por tanto, el sufrimiento (Villacieros, 2012, pág. 3).

La propuesta que se procura presentar permitirá conocer los diferentes tipos de duelo que se presentan en los estudiantes, al docente comprender las emociones, sentimientos y comportamiento del educando que experimenta en su diario vivir, posibilitando tomar acciones con el fin de contribuir en mejorar su rendimiento académico y comportamiento, por consiguiente: 
Podemos atravesar distintos procesos de duelo a lo largo de toda nuestra vida: desde la muerte de un ser querido, la ruptura con nuestra pareja o de aquel amigo de la infancia que de pronto se convierte en un desconocido, una mudanza, un cambio de trabajo o el niño que de pronto se da cuenta de que no es tan niño y que aquellos juegos que antaño le divertían ya no colman su alegría (Maura, 2014, pág. 4)

En el trascurrir de la vida, los seres humanos hacen frente a múltiples perdidas. Cabe indicar que no todas las pérdidas tienen igual significado ni interfieren de la misma forma en la vida diaria, pero toda pérdida con valor real o simbólico, conlleva un proceso de duelo, consciente o no, para quien lo sufre. Las pérdidas no solo se refieren a la muerte de un ser querido, también pueden sufrir pérdidas durante la vida de objetos que no tienen vínculo emocional, plantas o animales. Es evidente que el dolor experimentado dependerá del tipo de vínculo que una a aquello que se pierde y como afecte cada uno de los aspectos más relevantes de su vida.

Un estudiante que pase una etapa de duelo, podría verse afectado anímicamente, produciéndose perdidas de identidad, autoestima, siendo esto perjudicial para su interacción con sus compañeros, así como el rendimiento académico se vería mermado, siendo pertinente que docentes, padres, representantes, tengan conocimientos sobre el manejo asertivo del duelo en los estudiantes, con lo cual podrían contribuir en la aplicación de estrategias en favor de que el estudiante supere la etapa del duelo sin ver afectado en gran proporción su calidad educativa, Colomo Magaña (2016) plantea que "como docentes, tenemos la obligación de subsanar y cubrir las lagunas formativas respecto a la temática del duelo, tanto a nivel teórico (necesidad de conocer las principales consignas y factores a aplicar), como práctico" ( $p$. 73).

Educar desde una concepción pedagógica que contribuya a la inclusión de los estudiantes a una educación de calidad, es un reto, siendo un factor a tener en cuenta, el duelo, de ese modo, se tiene proyección de accionar en la conjugación holística de un estudiante en conformidad con su ser y medio social donde se interrelaciona, Vallejo Valdivieso, Zambrano Pincay, Vallejo Pilligua \& Bravo Cedeño (2019), comentan que 
se debe tener en cuenta una planificación educativa pensada en la diversidad de aptitudes para la potencialización de una educación en donde se tiene en cuenta las dimensiones del ser humano como un nexo complejo de interrelaciones, Páez (2018), comenta en este aspecto que la interrelación de los padres y representantes, contribuye a fortalecer la consolidación de lo planeado por el docente en apoyo de promover en el estudiante un crecimiento integral, en este caso de estudio desde el manejo del duelo. Un manejo adecuado del duelo, corresponde promover asertivamente las emociones, tarea para la cual el sistema educativo debe aplicar estrategias configuradas en razón de actuar en función de educar emocionalmente, siendo tarea de todos los actores educativos (Roque Díaz, 2018), siendo esta postura apoyada por Aparicio Albelo, González Rodríguez, Portal Denis, García Gómez, Machado Barberi \& Lorente Alguacil (2017), al indicar la importancia de educar emocionalmente a los estudiantes. Las emociones cuando no son manejadas asertivamente por parte de los estudiantes, puede afectar negativamente en su comportamiento, interacción y rendimiento académico (Pulido Acosta \& Herrera Clavero, 2017), siendo el duelo una emoción propia del ser humano, es pertinente que los docentes, padres y representantes, contribuyan en fomentar un adecuado manejo en el estudiante del mismo, esto podrá generar un ambiente de apoyo al estudiante en función de superar el duelo sin que este afecte profundamente sus deberes escolares, evitando la profundización de problemas en su natural interrelación social, de ese modo, la investigación tuvo como objetivo general determinar la relación entre el duelo familiar y el desarrollo emocional en los estudiantes de educación general básica superior y bachillerato general unificado de la Unidad Educativa Fiscal Cascol, de la Parroquia Cascol del Cantón Paján, provincia de Manabí, Ecuador. 
Revista Arbitrada Interdisciplinaria KOINONIA

Año V. Vol V. N9. Enero - Junio 2020

Hecho el depósito de Ley: FA2016000010

ISSN: 2542-3088

FUNDACIÓN KOINONIA (F.K). Santa Ana de Coro. Venezuela.

Carlos Julio Bastidas Martínez; Roberth Olmedo Zambrano Santos

\section{Aspectos teóricos referenciales de la investigación}

\section{El duelo}

En este sentido, Hoyos, (2015) afirma que "el duelo se puede definir como el conjunto de representaciones mentales y conductas vinculadas con una pérdida afectiva, cuyo objetivo es aceptar la realidad de la pérdida y adaptarse al nuevo entorno" (p.28), se trata de comprender que el duelo es una experiencia estresante pero normal, porque todas las personas deberá hacer frente a la pérdida de un objeto que represente valor sentimental o económico o la muerte de un familiar como afecta el desarrollo psicosocial, qué debe hacer ante esta situación, qué tiene que afrontar, por consiguiente ninguna persona puede evolucionar sin dolor, no puede crecer si no han experimentado cada una de las emociones y sensaciones antes descritas por cuanto permite lograr llegar a la autorrealización por cuanto los duelos son parte del crecimiento humano.

Por otra parte, Colomo (2016) considera que el duelo "se trata de un proceso psicológico complejo en el que se debe enfrentar el dolor de la ausencia, trabajando para reformular los vínculos afectivos con el fallecido con el fin de seguir viviendo tras asimilar la pérdida" (p.66). Siendo una respuesta cosmopolita a una pérdida (o a una separación) a la que se enfrentan los seres humanos de todas las edades y de todas las culturas. Además, este mismo autor precisa que el duelo como en todas las etapas de la vida afecta de una manera específica, debido que el dolor de la perdida se vuelve no solo es emocional sino también cognitiva, sobre todo en la edad adolescente, provocando en el mismo la pérdida del año escolar, pérdida de ganas de estar con amigos, inhibición de las tareas a realizar, las mismas que afectaran en su vida cotidiana a cada una de las personas.

Desde la perspectiva de Meza, García, Torres, Castillo, Sauri y Martínez (2008), el duelo es la reacción natural ante la pérdida de una persona, objeto o evento significativo; o también, la reacción emocional y de comportamiento en forma de sufrimiento y aflicción cuando un vínculo afectivo se rompe. En nuestra sociedad se tiende a pensar que el duelo se refiere a la muerte de un familiar, amigo, conocido o 
cualquier individuo pero también se produce como reacción ante una enfermedad, separaciones de los padres, bajas calificaciones, falta de empleo, perdida de la libertad, cambiar de domicilio, viajar a otro país, perdida de amistades. Lo esencial del duelo es que no depende de la naturaleza del objeto perdido, sino del valor que se le atribuye.

\section{Tipos de duelos}

\section{Duelo normal}

El duelo normal, es el más frecuente, se caracteriza por diferentes vivencias en todas las dimensiones de la persona incluye emociones y conductas que son normales después de una perdida.

El duelo es el conjunto de reacciones de tipo físico, emocional y social que se producen por el fallecimiento de una persona próxima y que pueden oscilar desde un sentimiento transitorio de tristeza hasta una sensación de desgarro y de desolación completa, que, en los casos más graves, puede durar años e incluso toda la vida (Martin, 2017, pág. 5).

El duelo normal tiene un tiempo de duración de seis meses se presenta sentimientos y conductas que a medida que pasa el tiempo, el dolor de la perdida disminuye. Entre las características primordiales que se dan está el desasosiego, dolor, culpa, rabia, sufrimiento, pérdida del sueño, peso, apetito; pérdida de confianza, sentimientos de inferioridad, pensamientos de muerte o conducta suicida.

\section{Duelo patológico}

También el Duelo patológico aparece de varias formas cuando las reacciones emocionales son muy intensas e impiden el funcionamiento en la vida diaria, y dura más de un año donde se presentan alucinaciones, ideas delirantes o pensamientos suicidad, ahí se puede hablar de duelo patológico. 
La imposibilidad de aceptar la pérdida, de adaptarse a su día a día y/o las emociones asociadas a ella, hacen que al recordar a su ser querido, lo haga desde el dolor y la angustia, no consiguiendo reubicar emocionalmente a su ser querido. Este dolor interfiere de forma significativa a nivel personal, familiar, relacional, laboral, espiritual, etc., e impide a la persona recordar a su ser querido desde lo positivo y seguir adelante con su vida (Sanchez, 2015, págs. 52-53).

En este sentido el duelo patológico se presenta con características de auto reproches, irritabilidad contra terceras personas, aislamiento social, descuido personal, consumo excesivo de alcohol, trastornos del sueño, dolores musculares, la persona se siente sin esperanza ni capaz de disfrutar de la vida.

\section{Duelo crónico}

El duelo crónico en las personas es cuando no se amolda a su nueva vida sigue sin aceptar la perdida de una persona, cosa, objeto. De forma explícita (Cabodevilla, 2007), expresa que:

El deudo se queda como pegado en el dolor, pudiéndolo arrastrar durante años, unido muchas veces a un fuerte sentimiento de desesperación. La persona es incapaz de rehacer su vida, se muestra absorbida por constantes recuerdos y toda su vida gira en torno a la persona fallecida, considerando como una ofensa hacia el difunto restablecer cierta normalidad (p. 170).

Es por ello que el duelo no evoluciona hacia su resolución y casi parece que el deudo mantiene a su ser querido vivo a través de su dolor. La duración de este duelo es excesiva y nunca llega a una conclusión satisfactoria, las personas que viven este tipo de duelo, se esfuerzan por vivir como vivían antes de la perdida pero no lo logran. Para esto deben estar consiente que las personas u objeto perdido no se recuperará, promover relaciones personales que le permitan al doliente cubrir alguna necesidad. 
Revista Arbitrada Interdisciplinaria KOINONIA

Año V. Vol V. N9. Enero - Junio 2020

Hecho el depósito de Ley: FA2016000010

ISSN: 2542-3088

FUNDACIÓN KOINONIA (F.K). Santa Ana de Coro. Venezuela.

Carlos Julio Bastidas Martínez; Roberth Olmedo Zambrano Santos

\section{Desarrollo emocional en el adolescente}

La adolescencia es un periodo crítico para el desarrollo social e individual en que los estudiantes enfrentan diversas situaciones que ponen en juego su inteligencia emocional que es un constructo que la última década ha tomado importancia para explicar el bienestar personal del educando en la familia, el colegio, en el trabajo. En el ámbito educativo es fundamental una adecuada relación positiva para encarar frustraciones, regular los estados de ánimo y controlar el miedo para dejar fluir la capacidad de pensar.

Mayer y Salovey (1997, citado en Ruiz, Fernández, Cabello y Extremera, 2006, p. 224), manifiesta que: "La IE se define como la capacidad para percibir, asimilar, comprender y regular nuestras emociones y las de los demás" (p.224). Se supone que los estudiantes con bajos niveles de inteligencia emocional presentan mayores niveles de impulsividad y un menor manejo de emoción el cual aumenta el riesgo de no poder sobrellevar el duelo por problemas familiares, divorcios o separaciones de sus padres, problemas con docentes, migración de algún familiar o enterarse que un familiar padece enfermedades catastróficas.

El desarrollo es un proceso de cambios que se producen en los individuos a través de toda su vida. Estos cambios pueden ser cualitativos o cuantitativos. Cualitativos se refiere a los cambios como la evolución del pensamiento, las emociones, las sensaciones, las habilidades, la atención, la memoria y cuantitativos se refieren a los cambios que pueden ser medidos la talla, el peso, etc.

Los adolescentes emocionalmente inteligentes tienen mejor salud física y psicológica y saben gestionar mejor sus problemas emocionales. En concreto, los estudios realizados informan de un menor número de síntomas físicos, menos niveles de ansiedad, depresión, ideación e intento de suicidio, somatización, atipicidad y estrés social, y una mayor utilización de estrategias de afrontamiento positivo para solucionar problemas (Extremera \& Fernandez, 2013, pág. 36). 
Para comprender las diferencias individuales en el desarrollo normal, se debe observar la vida de los individuos, características como: donde vive, a qué tipo de familia pertenece, economía del hogar, alimentación, acceso a salud, vivienda, educación, trabajo, el contexto en que se desarrolla el estudiante. Se debe trabajar de forma explícita los aspectos emocionales por el docente ya que es la única manera de crear una sociedad justa y equitativa, una sociedad con adolescentes creativos e inteligentes, pero sobre todo sano, integrado y feliz.

\section{MATERIALES Y MÉTODOS}

Se trabajó desde la perspectiva del enfoque cuantitativo, mediante un tipo de investigación correlacional transeccional, (Hernández, Fernández, Baptista, 2014), indican que este modalidad implica estudiar como una variable se relaciona con otra, teniéndose un diseño de campo no experimental, aplicándose en un único momento un cuestionario en escalamiento de Likert de cuatro alternativas de respuestas con 23 ítems, aplicándose una prueba piloto a 20 participantes con características similares a la población de estudio, pero que no pertenecen a la misma, obteniéndose un coeficiente de Alfa de Cronbach de 0,91 lo cual lo ubica en un rango de alta confiabilidad, complementándose su validez, mediante el veredicto de tres jueces expertos en el área de investigada, con la finalidad de indicar su contenido.

En referencia a la población de estudio, estuvo conformada por 200 estudiantes de educación general básica superior y bachillerato general unificado de la Unidad Educativa Fiscal Cascol, de la Parroquia Cascol del Cantón Paján, provincia de Manabí, Ecuador, la investigación se apoyó en la estadística descriptiva con el fin de describir el comportamiento de las variables de estudio, utilizándose el programa SPSS V 25 para el tratado de los datos tanto en la sección descriptivo como correlacional. 
Revista Arbitrada Interdisciplinaria KOINONIA

Año V. Vol V. N9. Enero - Junio 2020

Hecho el depósito de Ley: FA2016000010

ISSN: 2542-3088

FUNDACIÓN KOINONIA (F.K). Santa Ana de Coro. Venezuela.

Carlos Julio Bastidas Martínez; Roberth Olmedo Zambrano Santos

\section{RESULTADOS}

En este apartado, se procede a interpretar los resultados obtenidos de la aplicación del cuestionario para evaluar el duelo familiar y el desarrollo emocional:

\section{Cuadro 1}

Culpables por la separación de los padres

\begin{tabular}{l|r|r}
\multicolumn{1}{c}{ Ítems } & Estudiantes & \multicolumn{2}{c}{ Porcentaje } \\
\hline Siempre & 94 & $47 \%$ \\
\hline A veces & 75 & $37.5 \%$ \\
\hline Rara vez & 16 & $8 \%$ \\
\hline Nunca & 15 & $7.5 \%$ \\
\hline Total & 200 & $100 \%$
\end{tabular}

El $47 \%$ opina que siempre se sienten culpables por la separación de los padres, el $37.5 \%$ indica que a veces se siente culpable, un $8 \%$ manifiesta que rara vez se siente culpable y un $7.5 \%$ contestó que nunca se siente culpable.

\section{Cuadro 2}

Afecta la falta de comprensión de los padres y docentes

\begin{tabular}{l|r|r}
\multicolumn{1}{c}{ Ítems } & Estudiantes & Porcentaje \\
\hline Siempre & 188 & $94 \%$ \\
\hline A veces & 2 & $1 \%$ \\
\hline Rara vez & 3 & $1.5 \%$ \\
\hline Nunca & 7 & $3.5 \%$ \\
\hline Total & 200 & $100 \%$
\end{tabular}


Revista Arbitrada Interdisciplinaria KOINONIA

Año V. Vol V. No9. Enero - Junio 2020

Hecho el depósito de Ley: FA2016000010

ISSN: 2542-3088

FUNDACIÓN KOINONIA (F.K). Santa Ana de Coro. Venezuela.

Carlos Julio Bastidas Martínez; Roberth Olmedo Zambrano Santos

El $94 \%$ opina que siempre les afecta la falta de comprensión de los padres y docentes, la opción rara vez se conforma por el $1 \%$, rara vez lo constituye el $1.5 \%$ y nunca el $3.5 \%$

\section{Cuadro 3}

Afecta la separación de su novio/a

\begin{tabular}{c|r|r} 
Ítems & Estudiantes & \multicolumn{2}{c}{ Porcentaje } \\
\hline Siempre & 177 & $58.5 \%$ \\
\hline A veces & 56 & $28 \%$ \\
& 14 & $7 \%$ \\
\hline Rara vez & 13 & $6.5 \%$ \\
\hline Nunca & & $100 \%$
\end{tabular}

El $58.5 \%$ opina que siempre les afecta la separación de su novio/a, el $28 \%$ indica que a veces le afecta, un $7 \%$ manifiesta que rara vez le afecta y un $6.5 \%$ contestó que nunca le afecta.

\section{Cuadro 4}

Afectaría que uno de sus familiares migre a exterior por motivos económicos

\begin{tabular}{c|c|c} 
Ítems & Estudiantes & Porcentaje \\
\hline Siempre & 183 & $91.5 \%$ \\
\hline A veces & 9 & $4.5 \%$ \\
\hline Rara vez & 3 & $1.5 \%$ \\
\hline Nunca & 5 & $2.5 \%$ \\
\hline Total & 200 & $100 \%$
\end{tabular}


Revista Arbitrada Interdisciplinaria KOINONIA

Año V. Vol V. N9. Enero - Junio 2020

Hecho el depósito de Ley: FA2016000010

ISSN: 2542-3088

FUNDACIÓN KOINONIA (F.K). Santa Ana de Coro. Venezuela.

Carlos Julio Bastidas Martínez; Roberth Olmedo Zambrano Santos

El 91.5\% opina que siempre podría afectarle la migración familiar, el $4.5 \%$ indica que a veces le podría afectar, un 1.5\% manifiesta que rara vez le afectaría y un $2.5 \%$ contestó que nunca le afectaría.

\section{Cuadro 5}

Relación entre el duelo familiar y el desarrollo emocional en los estudiantes

\begin{tabular}{|c|c|c|c|}
\hline & & DF & $\mathrm{DE}$ \\
\hline \multirow[t]{3}{*}{ DF } & Correlación de Pearson & 1 &,$- 232^{* \star}$ \\
\hline & Sig. (bilateral) & & 0,001 \\
\hline & $\mathrm{N}$ & 200 & 200 \\
\hline \multirow[t]{3}{*}{$\mathrm{DE}$} & Correlación de Pearson &,$- 232^{* *}$ & 1 \\
\hline & Sig. (bilateral) & 0,001 & \\
\hline & $\mathrm{N}$ & 200 & 200 \\
\hline
\end{tabular}

El resultado de -,232 indica una correlación de Pearson negativa débil, lo cual implica una relación inversamente proporcional, es decir que el desarrollo emocional de los estudiantes incide directamente en el afrontamiento del duelo familiar. Al existir correlación significativa en el nivel 0,01 se acepta la hipótesis afirmativa y se rechaza la nula. En este sentido:

\section{Prueba de hipótesis}

Afirmativa: Existe relación significativa entre el duelo familiar y el desarrollo emocional en los estudiantes.

Negativa: No existe relación significativa entre el duelo familiar y el desarrollo emocional en los estudiantes.

Se acepta la hipótesis afirmativa y se rechaza la negativa. 
Revista Arbitrada Interdisciplinaria KOINONIA

Año V. Vol V. N9. Enero - Junio 2020

Hecho el depósito de Ley: FA2016000010

ISSN: 2542-3088

FUNDACIÓN KOINONIA (F.K). Santa Ana de Coro. Venezuela.

Carlos Julio Bastidas Martínez; Roberth Olmedo Zambrano Santos

\section{DISCUSIÓN}

La dimensión culpable por la separación de los padres, las opciones negativas corresponden al $84,5 \%$ de la población total, indicando que existe un elevado número de estudiantes que manifiestan culpabilidad por la separación de sus padres, lo cual es perjudicial por cuanto la separación trae consigo duelo, aunado a pensamientos donde se asume una culpa que generalmente no causada por el hijo o hija. Alomo, Muraro, Gurevicz, Castro Tolosa \& Lombardi (2016), explican que el sentimiento de culpa, puede generar un "masoquismo primario", siendo esta una aptitud aversiva en el estudiante por cuanto podría complicar la aparición de conductas no apropiadas en detrimento de calidad de vida emocional y educativa de la persona.

En cuanto a la dimensión afecta la falta de comprensión de los padres y docentes, el $94 \%$ de la población manifiesta que se ve afectada, lo cual produce un quiebre en la relación con los padres y docentes, (Razeto, 2016) considera la necesidad de que la familia se involucre en las actividades propias de los estudiantes, esto le permitirá mejorar su socialización y rendimiento académico, especialmente la comprensión lectora, lo cual indica que un estudiante que se perciba como no comprendido por sus padres y docentes, podría verse retraído o rebelde para el cumplimiento de sus deberes escolares, aunado a la posibilidad de condicionar esta conducta negativa a otras áreas de sus vidas.

La dimensión afecta la separación de su novio/a, el 86,5 opina que se ve afectado/a emocionalmente, con sentimientos de culpabilidad y duelo, (Barajas Márquez \& Cruz del Castillo, 2017), comentan el rol fundamental que tiene el noviazgo para el ser humano, destacando que la ruptura afecta considerablemente la interrelación del afectado/a consigo mismo y su entorno, siendo esta una situación que debe ser apoyada por padres y docentes con la finalidad de que los estudiantes tengan el apoyo emocional pertinente para superar el duelo.

La dimensión afectaría que uno de sus familiares migre a exterior por motivos económicos, se destaca que el $91,5 \%$ de los encuestados manifiesta que la migración familiar podría ocasionarles duelo, afectando su interrelación social, así como su 
efectividad académica, (Lotero-Echeverri \& Pérez-Rodríguez, 2019), consideran que la migración trae consigo cambios estructurales tanto en el país de salida como de llegada del migrante, aunado a la afectación psicológica causada por la separación del proceso migratorio donde las familias ven partir a sus seres queridos, siendo esta situación concomitante con la perspectiva de los estudiantes encuestados que perciben duelo por causa de sus familiares migrantes.

Al aceptarse la hipótesis afirmativa, se comprueba que existe relación entre el duelo familiar y las emociones de los estudiantes, a juicio de (García-Viniegras, Grau Abalo \& Pedreira, 2014), indican que el impacto del duelo es un factor de riesgo importante para la salud, por lo tanto, es necesario generar un abordaje primario del mismo con la finalidad de minimizar sus consecuencias en la persona, siendo esta una actividad que los padres y docentes deben asumir con la finalidad de contribuir a una mejor educación desde la calidad de vida de los estudiantes, siendo necesaria la efectiva aplicación de estrategias para el manejo asertivo del duelo en los estudiantes.

\section{CONCLUSIONES}

El duelo es un proceso normal de adaptación de las personas ante las pérdidas permitiéndole enfrentarse y ajustarse al medio en que se desarrolla. En definitiva el duelo familiar requiere más evidencia, para así entonces, saber cómo lidiar con un suceso tan traumático como este. Esto se ve acentuado en el país, donde la investigación es aun deficiente, fomentando que la población siga desconociéndolo y tratándolo como un duelo de perder un familiar o allegado.

Estamos llamados a cumplir un rol fundamental en la difusión, análisis del duelo en la sociedad ecuatoriana orientando e interviniendo lo necesario para que los docentes, padres de familia y sociedad en general conozca del duelo familiar y como les afecta en el desarrollo emocional del educando. Se debe empoderar acerca de esta realidad social que cambia y requiere equilibrio, armonía. Para esto el docente debe conocer de neuro semántica para que pueda darle un significado positivo a la muerte de un familiar, 
Revista Arbitrada Interdisciplinaria KOINONIA

Año V. Vol V. N9. Enero - Junio 2020

Hecho el depósito de Ley: FA2016000010

ISSN: 2542-3088

FUNDACIÓN KOINONIA (F.K). Santa Ana de Coro. Venezuela.

Carlos Julio Bastidas Martínez; Roberth Olmedo Zambrano Santos

conocido, a un divorcio, a una enfermedad crónica o a un despido del trabajo o problemas con padres o docentes.

El proceso de duelo puede convertirse en aprendizaje transcendental, pues la experiencia dolorosa implica un nuevo conocimiento de sí mismo, dejando enseñanzas útiles para el desarrollo de la vida. Las personas, utilizan diversos mecanismos para afrontar el dolor tras la pérdida, ellos permiten restaurar la vida hacia etapas de mayor satisfacción y espacios más edificantes en el proceso de vida. Nada se acaba cuando una relación termina, cuándo uno viaja, cuando se separa, cuando nos diagnostica una enfermedad grave, en definitiva volver a vivir, es posible.

El docente debe conocer de neuro semántica para que pueda darle un significado positivo a la muerte de un familiar, conocido, a un divorcio, a una enfermedad crónica o a un despido del trabajo o problemas con padres o docentes.

\section{FINANCIAMIENTO}

No monetario.

\section{AGRADECIMIENTOS}

A los estudiantes de educación general básica superior y bachillerato general unificado de la Unidad Educativa Fiscal Cascol, de la Parroquia Cascol del Cantón Paján, provincia de Manabí, por apoyar la investigación.

\section{REFERENCIAS CONSULTADAS}

Atxotegui, J. (2010). Los duelos de la migraciòn: una aproximacion psicopatològica y psicosial. [The duels of migration: a psychopathological and psychosial approach]. Recuperado de https://n9.cl/3gz45

Alomo, Martín; Muraro, Vanina; Gurevicz, Mónica; Castro Tolosa, Silvana; Lombardi, Gabriel. (2016). El sentimiento inconsciente de culpa freudiano: clínica diferencial y suposición de sujeto. Una aproximación metodológica. [Freudian unconscious feeling of guilt: differential clinic and subject assumption]. Anuario de Investigaciones, vol. XXIII, 2016, pp. 15-21. Recuperado de https://www.redalyc.org/pdf/3691/369152696038.pdf 
Revista Arbitrada Interdisciplinaria KOINONIA

Año V. Vol V. N9. Enero - Junio 2020

Hecho el depósito de Ley: FA2016000010

ISSN: 2542-3088

FUNDACIÓN KOINONIA (F.K). Santa Ana de Coro. Venezuela.

Carlos Julio Bastidas Martínez; Roberth Olmedo Zambrano Santos

Aparicio Albelo, A., González Rodríguez, L., Portal Denis, N., García Gómez, N., Machado Barberi, F., \& Lorente Alguacil, L. (2017). Educar la inteligencia emocional de los estudiantes de Logofonoaudiología en la comunidad universitaria. [Educate the emotional intelligence of Logophonoaudiology students in the university community]. EDUMECENTRO, 9(1), 161-174. Recuperado de http://www.revedumecentro.sld.cu/index.php/edumc/article/view/890

Barreto, P., Torre, O. y Pérez, M. (2012). Detección de duelo complicado. [Difficult duel detection]. Psicooncologia, 9(2-3), 355-368. DOI: 10.5209/rev PSIC.2013.v9.n23.40902.

Barajas Márquez, Miriam W.; Cruz del Castillo, Cinthia (2017). Ruptura de la pareja en jóvenes: factores relacionados con su impacto Enseñanza e Investigación . [Young couple's breakup: factors related to its impact] en Psicología, vol. 22, núm. 3, septiembre-diciembre, 2017, pp. 342-352. Recuperado de https://www.redalyc.org/pdf/292/29255775008.pdf

Cabodevilla, I. (2007). Las pèrdidas y sus duelos. [Losses and their duels. Annals of Navarra Health System, 30 (3), 170]. Anales del Sistema Sanitario de Navarra, 30(3), 170.

Cadena, P., Rendón, R., Aguilar, J., Salinas, E., Cruz, F., y Sangerman, D. (2017). Métodos cuantitativos, métodos cualitativos o su combinación en la investigación: un acercamiento en las ciencias sociales. [Quantitative methods, qualitative methods or their combination in research: an approach in the social sciences]. Scielo, 8(7), $1603-1617$.

Colomo Magaña, E. (2016). Pedagogía de la Muerte y Proceso de Duelo. Cuentos como Recurso Didáctico. [Pedagogy of Death and Grief Process. Tales as a Teaching Resource]. REICE. Revista Iberoamericana Sobre Calidad, Eficacia Y Cambio En Educación, 14(2). http://dx.doi.org/10.15366/reice2016.14.2.004

Extremera, N., \& Fernandez, P. (2013). Inteligencia Emocional en adolescentes. [Emotional Intelligence in adolescents, parents and teachers (352), 34-39]. Padres y Maestros(352), 34-39. Recuperado de https://n9.cl/s19v

Gaeta, V. (2015). Desarrollo Psicosocial del adolescente. [Desarrollo Psicosocial del adolescente]. Revista Chilena de Pediatría, 86(6), 436-443. Recuperado de https://n9.cl/r96kz

Garciandía, J. (2013). Familia, suicidio y duelo. [Family, suicide and grief]. Revista Colombiana de Psiquiatría, 43(1), 71-79. Recuperado de https://n9.cl/4hws 
Revista Arbitrada Interdisciplinaria KOINONIA

Año V. Vol V. N9. Enero - Junio 2020

Hecho el depósito de Ley: FA2016000010

ISSN: 2542-3088

FUNDACIÓN KOINONIA (F.K). Santa Ana de Coro. Venezuela.

Carlos Julio Bastidas Martínez; Roberth Olmedo Zambrano Santos

García-Viniegras, Carmen Regina Victoria, Grau Abalo, Jorge A, \& Pedreira, Infante. (2014). Duelo y proceso salud-enfermedad en la Atención Primaria de Salud como escenario para su atención. [Grief and health-disease process in Primary Health Care as a setting for their care]. Revista Cubana de Medicina General Integral, 30(1), 121-131. Recuperado de https://n9.cl/x6aw

Guillén, E., Gordillo, M., Gordillo, D., Ruiz, I., y Gordillo, T. (2013). Crecer con la pérdida: El duelo en la infancia y adolescencia. [Growing up with loss: Grief in childhood and adolescence]. INFAD Revista de Psicología, 2(1), 493-498. Recuperado de https://n9.cl/e42ji

Hernández, Roberto, Fernández, Carlos, Baptista, Pilar (2014). Metodología de la Investigación. [Investigation methodology]. (sexta edición). Editorial Mc - Graw Hill Interamericana. México.

Hoyos, M. (2 de Febrero de 2015). ¿Entendemos los adultos el duelo de los niños? [Do we as adults understand the grief of children?]. ACTA PEDIÁTRICA, 73(2), 28. Recuperado de https://n9.cl/k971h

Lotero-Echeverri, G., y Pérez-Rodríguez, M.A. (2019). Migraciones en la sociedad contemporánea: Correlación entre migración y desarrollo. [Migrations in contemporary society: Correlation between migration and development. Challenges]. Retos Revista de Ciencias de la Administración y Economía, 9(17), 145-159. https://doi.org/10.17163/ret.n17.2019.09

Márquez, X. (2005). Ni contigo ni sin tì: la pareja irrompible. [Neither with you nor without you: the unbreakable couple]. Revista Interconental de Psicologia y Educacion, 7(2), 27-42. Recuperado de https://www.redalyc.org/pdf/802/80270203.pdf

Martin, M. (2017). El papel de enfermeria en el duelo de familiares de pacientes terminales (Tesis de pregrado). [The role of nursing in the grief of relatives of terminally ill patients (Undergraduate thesis)]. Recuperado de https://n9.cl/6i5ml

Martos, I. Sanchez, M., y Guedes, Ch. (2016). Duelo por muerte perinatal, un duelo desautorizado. [Mourning for perinatal death, an unauthorized mourning]. Revista Española de comunicación en salud, 7(2), 300-309. Recuperado de https://n9.cl/43nv

Martínez, M. (2006). La investigación cualitativa (Síntesis conceptual). [Qualitative research (Conceptual synthesis)]. Dialnet, 9(1) ,123-131. Recuperado de https://n9.cl/dns2 
Revista Arbitrada Interdisciplinaria KOINONIA

Año V. Vol V. N9. Enero - Junio 2020

Hecho el depósito de Ley: FA2016000010

ISSN: 2542-3088

FUNDACIÓN KOINONIA (F.K). Santa Ana de Coro. Venezuela.

Carlos Julio Bastidas Martínez; Roberth Olmedo Zambrano Santos

Maura, M. (2014). Duelo y Apego: De la creación del vínculo a la pérdida del mismo.(Tesis de grado). [Grief and Attachment: From the creation of the link to the loss of it. (Degree thesis)]. Recuperado de https://n9.cl/6wcy

Meza, E., García, S., Torres, A., Castillo, L., Sauri, S. y Martínez, B. (2008). El proceso del duelo. Un mecanismo humano para el manejo de las pérdidas emocionales. [The grieving process. A human mechanism for managing emotional losses]. Revista de Especialidades Médico-Quirúrgicas, 13(1), 28-31 Recuperado de https://n9.cl/sapi

Pascual, A. (2016). El duelo ante la muerte en la infancia y adolescencia (Tesis de grado). Universidad de la Rioja, España. [Grief over death in childhood and adolescence (Bachelor thesis)]. Recuperado de https://n9.cl/kjh9

Páez, A. (2018). Docentes y padres en el proceso de aprendizaje de los estudiantes. [Teachers and parents in the student learning process]. EPISTEME KOINONIA, 1(2), 18-34. Recuperado de https://n9.cl/o201

Pereira, R. \& Vannnotti, M. (2011). Un caso de duelo familiar. [A case of family grief. Research gate]. Researchgate. Recuperado de https://n9.cl/pkqbr

Pulido Acosta, F. \& Herrera Clavero, F. (2017). La influencia de las emociones sobre el rendimiento académico. [The influence of emotions on academic performance]. Recuperado de http://dx.doi.org/10.22235/cp.v11i2.1344

Ramìrez, M. (2007). Los Estados emocionales en los momentos de duelo y la forma de abordarlos. [Emotional states in moments of grief and how to deal with them]. Ciencia y Cuidado, 4(4), 36-44.

Razeto, Alicia. (2016). El involucramiento de las familias en la educación de los niños: Cuatro reflexiones para fortalecer la relación entre familias y escuelas. [The involvement of families in the children's education: Four reflections to strengthen the relationship between families and schools]. Páginas de Educación, 9(2), 184201. Recuperado de 2020, de https://n9.cl/vrkuj

Roque Díaz, R. (2018). Desarrollo personal y manejo asertivo de emociones en estudiantes. [Personal development and assertive management of emotions in students]. EPISTEME KOINONIA, 1(2), 61-82. Recuperado de https://n9.cl/lbwvv 
Revista Arbitrada Interdisciplinaria KOINONIA

Año V. Vol V. N9. Enero - Junio 2020

Hecho el depósito de Ley: FA2016000010

ISSN: 2542-3088

FUNDACIÓN KOINONIA (F.K). Santa Ana de Coro. Venezuela.

Carlos Julio Bastidas Martínez; Roberth Olmedo Zambrano Santos

Ruiz, D., Fernández, P., Cabello, R. \& Extremera, N. (2006). Inteligencia Emocional Percibida y consumo de tabaco y alcohol en adolescentes. [Perceived Emotional Intelligence and tobacco and alcohol consumption in adolescents. Anxiety and Stress, 12 (2-3), 223-230]. Ansiedad y Estrés, 12(2-3), 223-230. Recuperado de https://n9.cl/7dmq2

Sánchez, Ana. (2018). Etapa de duelo en los adolescentes de educación básica superior de la Unidad Educativa Nelson Isauro Torres de la ciudad de Cayambe en el año lectivo 2017-2018 (Tesis de pregrado). [Adolescents of higher basic education's mourning state at Nelson Isauro Torres Educational Unit in the city of Cayambe in the academic year 2017-2018 (Undergraduate thesis)]. Universidad Central del Ecuador, Quito, Ecuador. Recuperado de https://n9.cl/8ud6

Sanchez, J. (2015). Eficacia de un Protocolo de intervenciòn psicològica en procesos de duelo patològico (Tesis doctoral). [Efficacy of a protocol for psychological intervention in pathological grief processes (doctoral thesis)]. Recuperado de https://n9.cl/fy9oi

Vallejo Valdivieso, P., Zambrano Pincay, G., Vallejo Pilligua, P., \& Bravo Cedeño, G. (2019). Modelos de planificación educativa y diversidad en aulas de clases. [Models of educational planning and diversity in classrooms]. CIENCIAMATRIA, 5(9), 302-315. https://n9.cl/vxpj4

Vilajoana, J. (2017). Duelo anticipado en familiares cuidadores e enfermos de Alzheimer $y$ otras demencias. [Early mourning in family caregivers of patients with Alzheimer's disease and other dementias]. Recuperado de https://n9.cl/pbrm

Villacieros, M., Magaña, M., Bermejo, J., Carabias, R. y Serrano, I. (2012). Estudio del perfil de una población de personas en duelo complicado que acuden a un centro de escucha de duelo. [Study of people population's profile in complicated mourning who come to a mourning listening center]. Med Paliat, 1-7 http://dx.doi.org/10.1016/j.medipa.2012.05.007 